\title{
PLACENTAL PATHOLOGY IS RELATED TO POSTNATAL CIRCULATORY COMPROMISE IN PRETERM INFANTS
}

\author{
D.C. Vijlbrief ${ }^{1}$, M.J.N.L. Benders ${ }^{1}$, H. Kemperman ${ }^{2}$, F. van Bel ${ }^{1}$, W.B. de $\operatorname{Vries}^{1}$ \\ ${ }^{1}$ Neonatology, ${ }^{2}$ Clinical Chemistry and Hematology, University Medical Center Utrecht, Utrecht, The \\ Netherlands
}

Background: Impairment of gas exchange and blood flow through the placenta leads to hypoxia and hypercapnia, causing increased systemic vascular resistance and tachycardia, influencing the cardiovascular system of the fetus. The biomarker B-type natriuretic peptide (BNP) can be used to identify significant cardiovascular disease in infants.

Objective: To investigate whether placental pathology was associated with significant cardiovascular compromise in the premature infant as diagnosed by elevated BNP-levels after birth.

Methods: From October 2009 until October 2010134 infants born with GA<32 weeks were evaluated. Placenta pathology was performed and related to BNP levels at admission, within 6 hours after birth.

Results: Chorioamnionitis was present in $32 \%$, signs of pathologic increased maturation in $55,2 \%$. Increased placental maturation showed strong correlation with clinical diagnosis of pre-eclampsia $(\mathrm{PE})(\mathrm{r}=0.59, \mathrm{p}<$ 0.001). BNP-levels were elevated in pregnancies complicated by clinical or pathological signs of PE (median $56 \mathrm{p} / \mathrm{mol} / \mathrm{l}$ vs. $106 \mathrm{p} / \mathrm{mol} / \mathrm{L} ; \mathrm{p}<0.05)$. The highest BNP-levels were found in those infants where PE was complicated by fetal distress $(91 \mathrm{pmol} / \mathrm{L}$ vs. 390pmol/L; $<<0.01)$. The presence of chorioamnionitis was associated with lower BNP-levels, although this was not statistically significant $(55 \mathrm{pmol} / \mathrm{L}$ vs. $94 \mathrm{pmol} / \mathrm{L} ; \mathrm{p}=$ 0.12 ) and possibly related to $\mathrm{PE}$ in the other group.

Conclusion: This study shows that those infants, where maternal, pathological or clinical, PE was complicated by signs of fetal distress, are the most likely to have cardiovascular compromise at birth. Whether this is directly related to fetal cardiovascular adaptation to placental vascular pathology needs further investigation. 